\title{
(Con)viviendo con malaria: salud, enfermedad y autoatención en una comunidad rural en la Amazonia brasileña
}

\author{
DASSUEM NOGUEIRA Y LUIZA GARNELO
}

DASSUEM NogueIRA

Instituto Leônidas \& Maria Deane, Fundação Oswaldo Cruz, Manaos,

Amazonas, Brasil

dassuem.nogueira@gmail.com

LUIZA GARNELO

Instituto Leônidas \& Maria Deane,

Fundação Oswaldo Cruz, Manaos,

Amazonas, Brasil

malupereira2011@gmail.com

Traducción: Juana Valentina Nieto Moreno
En el artículo se analiza el sistema de autoatención a la malaria en una localidad rural de la Amazonia brasileña. Se explora la atribución de significados a esta enfermedad, sus causas, sintomatología, tratamientos, prevención y consecuencias para la salud y la capacidad laboral. Se describen estrategias para lidiar con portadores de malaria interna - cuando la persona padece malaria, pero los síntomas son poco evidentes y no se establece el papel social de enfermo-. A partir de casos socialmente definidos como malaria y malaria interna se instituye el manejo de relaciones intracomunitarias de poder que pretenden garantizar recursos provenientes de políticas sanitarias y de la ayuda social a la población considerada en riesgo de enfermarse.

PALABRAS CLAVE: autoatención, atención primaria a la salud, salud rural, malaria, antropología de la salud

Living with Malaria: Health, Illness and Self-attention in a Rural Community in the Brazilian Amazon

The article analyzes the system of self-attention for malaria in a rural town in the Brazilian Amazon. Exploits the assignment of meanings to this disease, its causes, symptomatology, treatment, prevention and consequences for health and capacity for work. The characteristics of the self-attention system include differentiated strategies to deal with internal malaria, state of liminality in which a person is classified as a carrier of malaria, but the symptoms are still not evident and the social role of the sick person is not established. From the socially defined cases such as malaria and internal malaria, the management of intra-community power relations is also established, which seeks to guarantee resources originating from health policies and social assistance.

KEYWORDS: self-attention, primary health care, rural health, malaria, anthropology of health 


\section{Introducción}

a malaria es una enfermedad infecciosa, producida por protozoarios del gé-

nero Plasmodium, transmitida por la hembra infectada del mosquito de género Anopheles. Su incidencia es definida por el índice parasitario anual (IPA). La fórmula del IPA — número anual de casos/población susceptible $\times 1000$ — también permite clasificar las áreas de riesgo de transmisión, riesgo alto > 49.9 casos/1 000; riesgo mediano entre 10 y 49.8/1 000 (Ministério da Saúde, 2003). Brasil es el país con más casos de malaria en América y la Amazonia es la región brasileña con mayor riesgo de contracción de la enfermedad, con 94.9\% de los casos (Ministério da Saúde, 2015; OPS y OMS, 2018).

A pesar de la reciente tendencia a la reducción de los índices de malaria, su disminución no es uniforme y casi 46 municipios amazónicos mantienen un IPA muy elevado (Ministério da Saúde, 2015). Éste es el caso de Manaos, capital del estado de Amazonas, que alterna periodos de alto riesgo — IPA de $1659.7 / 1000$ en 2005 (Saraiva et al., 2009)—, ${ }^{1}$ con una oscilación en el número de casos — 5541 en 2014; 8530 en 2015- y una reducción de las tasas observadas durante el año en el que el control de la enfermedad se mostró más eficaz — IPA 4.6/1000 en 2016-, que tienden a elevarse si éste se interrumpe (Ministério da Saúde, 2017). El área rural de Manaos presentó 33.2\% de los casos ocurridos en la capital (Saraiva et al., 2009), lo que produce un impacto negativo en las condiciones de vida de su población (Ministério da Saúde, 2015; Canelas, Castillo-Salgado y Ribeiro, 2018).

La alta incidencia de la malaria está relacionada con situaciones de vulnerabilidad ambiental y socioeconómica que favorecen la generación de criaderos, la reproducción del vector y la elevada exposición de las personas a los parásitos. Las

$1 \quad$ Cuando el IPA rebasa la proporción de 1000, indica que esas personas han padecido malaria más de una vez ese año. 
limitaciones de acceso al diagnóstico y tratamiento precoz también contribuyen a la persistencia del cuadro. El predominio de un modelo de vivienda rural con ausencia completa o parcial de paredes también acentúa la exposición humana a las picaduras de mosquitos vectores (Braz, Duarte y Tauil, 2013; Katsuragawa et al., 2008).

El mantenimiento de altos índices de malaria y la recurrencia de su perfil endémico-epidémico en la población están asociados de manera íntima a las situaciones de vulnerabilidad social, económica y ambiental que enfrentan quienes viven en los espacios rurales amazónicos (Canelas et al., 2018). Se trata de poblaciones que conviven de manera intensa y prolongada con la malaria. Por esta razón, han desarrollado concepciones singulares sobre la enfermedad y los modos de lidiar con ella, así como sobre los servicios y profesionales de salud que actúan en ese campo.

Menéndez propuso el concepto de autoatención, elemento clave para la discusión que aquí se plantea, y lo definió como sigue:

Las representaciones y prácticas que la población utiliza a nivel de sujeto y grupo social para diagnosticar, explicar, atender, controlar, aliviar, aguantar, curar, solucionar o prevenir los procesos que afectan su salud en términos reales o imaginarios, sin la intervención central, directa e intencional de curadores profesionales, aun cuando éstos pueden ser la referencia de la actividad de autoatención (2003: 198).

También señala que, aunque de carácter autónomo, las producciones socioculturales instituidas y llevadas a cabo en prácticas de autoatención no excluyen los conocimientos biomédicos. Por el contrario, éstos circulan en el tejido social y son apropiados. Ofrecen elementos de significación reinterpretados por los productores de autoatención y utilizados según sus necesidades y prioridades. No es raro que modelos explicativos y técnicas consideradas superadas y descartadas por el modelo médico hegemónico tengan una amplia circulación entre los practicantes de la autoatención, en particular los de estratos sociales populares.

Este artículo analiza cómo los habitantes de una localidad rural de la Amazonia brasileña, interpretan, viven y producen saberes y prácticas sobre la enfermedad por malaria y sus cuidados, en interfaz con los servicios oficiales de salud encargados de la prevención y cuidados dirigidos a esta endemia.

\section{Contexto de la investigación}

Nuestra Señora del Livramento, por lo regular denominada Livramento, es un asentamiento localizado a orillas del Rio Negro, a unos $25 \mathrm{~km}$ de Manaos —latitud 3'1'45.38', longitud 60'10'48.01'”, al cual se accede sólo por vía fluvial. No se encontraron publicaciones anteriores que hablen de la comunidad de Livramento.

Ahí vive una población mestiza, conocida como cabocla, de 250 familias. Buena parte desciende de grupos indígenas que emigraron de otras partes del valle amazónico, en particular del Alto Rio Negro, que se mezclaron con una población regional. Su mestizaje remoto no permite una identificación étnica específica (Adams, Murrieta y Neves, 2006). Entre las consecuencias del mestizaje se observa la persistencia de una cosmología un tanto vaga, que no se vincula a una etnia en particular pero permanece como un elemento afectivo-cognitivo importante que orienta las relaciones de los humanos entre sí y con la naturaleza, y subyace en gran parte de las explicaciones sobre la causalidad de las enfermedades y las estrategias para tratarlas (Garnelo y Sampaio, 2005).

Estas singularidades históricas y sociales contribuyen al mantenimiento de las características rurales y singulares de Livramento, y suavizan la influencia de los modos de vida urbanos de la vecina 


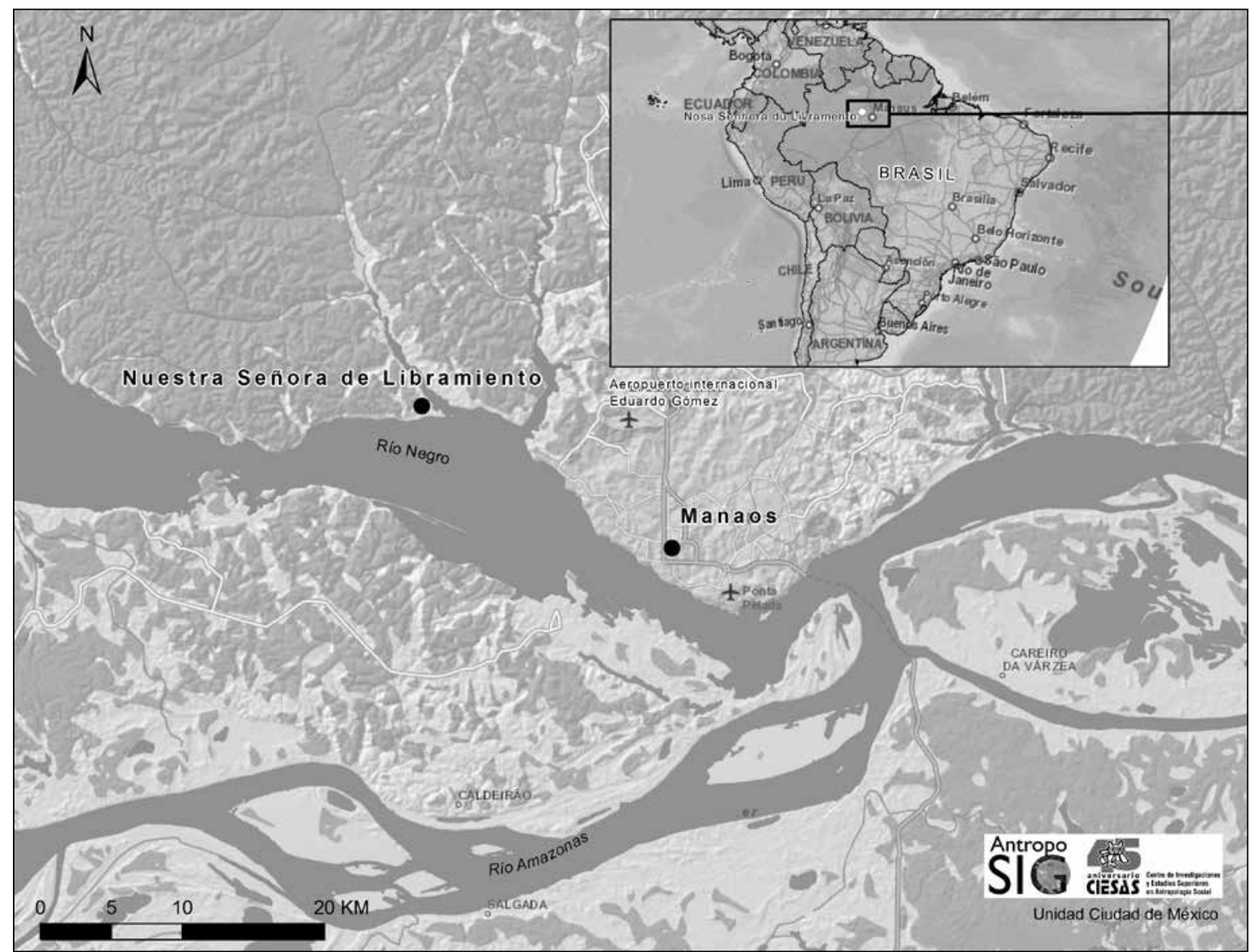

Elaborado por Bulmaro Sánchez Sandoval.

Manaos, una metrópoli regional de casi dos millones de habitantes, en acelerado proceso de globalización de las relaciones económicas y sociales.

Livramento surgió en la confluencia de un pequeño caudal de agua conocido como igarapé Tarumã Mirim, con el gran Rio Negro, hacia 1960. Fue formada por migrantes que buscaban las facilidades derivadas de vivir cerca de una gran ciudad como Manaos, pero sin abandonar el modo de vida rural. En 2004, las tierras situadas a la orilla derecha del igarapé Tarumã Mirim se incorporaron a la Reserva de Desarrollo Sostenible del Tupé (RDSTupé). Esto alteró a profundidad los modos de vida de los habitantes de Livramento, que comenzaron a enfrentar restricciones a sus actividades de subsistencia, como la prohibición de talar árboles para la siembra de cultivos y la limitación de la pesca, lo que los obligó a adquirir gran parte de sus productos alimenticios en el comercio de Manaos.

En el periodo de recolección de datos ya se habían modificado las actividades laborales, pues las principales fuentes de ingresos de antes, como la extracción de madera y la fabricación de carbón, ya se consideraban crímenes ambientales. La siembra estaba permitida, siempre que se autorizara mediante licencia ambiental. En la práctica, el atraso de las 
autorizaciones impedía que los habitantes obtuvieran la licencia ambiental a tiempo para efectuar la secuencia de operaciones de tumba, siembra y recolección agrícola, de acuerdo con la temporalidad y ritmos marcados por las estaciones del año.

La reducción del abanico de actividades productivas autorizadas erosionó la capacidad de generación de ingresos de las familias. En consecuencia, la principal fuente de ingresos pasó a ser el programa de transferencia condicional de renta, en Brasil llamado Bolsa Familia. Pocas familias añadían el ingreso de las rentas a la jubilación y los salarios de un pequeño número de funcionarios públicos que laboraba en los establecimientos escolares y de salud.

Los datos de la Fundación de Vigilancia en Salud del Amazonas (Braz, Duarte y Tauil, 2013) señalan que la malaria destacaba como uno de los grandes problemas de salud de la localidad, aunque coexistiera con otros problemas sociosanitarios también graves, como la precariedad de las condiciones de vivienda y la falta de saneamiento y de condiciones mínimas de urbanización, los cuales favorecían la formación de criaderos en los que proliferaban los mosquitos vectores de la malaria.

La investigación incluyó un periodo intensivo de cinco meses, a los que habría que añadir los diez años de trabajo previo de Luiza Garnelo con esta comunidad. Las técnicas utilizadas para la recopilación de datos fueron la observación participante en la interacción cotidiana con familias residentes en Livramento, con énfasis particular en situaciones que involucraban la enfermedad y el tratamiento de casos de malaria. También se contempló la participación en eventos sociales y reuniones en los que se discutían los problemas de la localidad, en particular aquellos en los que se hablaba de la malaria. La recolección de datos se complementó con entrevistas a 18 miembros de la comunidad, que suman 56 horas de grabación. Las entrevistas con los actores clave - líderes comunitarios, habitantes y promotores de salud- contenían preguntas guía que buscaban aprovechar la experiencia de los residentes con malaria, ya fuera en la condición de enfermos o en el cuidado de familiares o comensales enfermos de malaria.

Más allá de ser un fenómeno biológico, la enfermedad debe entenderse como un proceso social capaz de movilizar al mismo tiempo las esferas biológicas, sociales, simbólicas, afectivas y políticas para superar la situación de enfermedad y retomar el estado de salud (Buchillet, 1991).

Entre las estrategias utilizadas en Livramento para lidiar con la malaria, destaca la autoatención en sentido amplio que, de acuerdo con Menéndez:

No se restringe a las prácticas dirigidas a la búsqueda de la curación, sino que comporta también un amplio abanico de acciones dirigidas a la reproducción de la vida, incluidos los cuidados con los niños, las niñas y las gestantes, con el agua para beber y cocinar, la limpieza del lugar, la selección y la distribución de los alimentos e incluso la atención dada a los muertos (2003: 199).

Estas estrategias promueven la coexistencia de modelos explicativos del proceso salud y enfermedad, que incluyen el modelo biomédico hegemónico, apropiado, probado y reinterpretado para ganar coherencia con la realidad social y las técnicas y otros modos de cuidado accesibles al grupo productor de la autoatención.

\section{(Con)viviendo con la malaria en Livramento}

Los habitantes de Livramento se exponen a diario a informaciones biomédicas sobre la malaria, pues los servicios de control de la enfermedad actúan en esta localidad desde su fundación, como consecuencia del reconocimiento público de que se encuentra en una importante área endémica de la Amazonia brasileña.

La familiaridad de la población con las informaciones sobre las relaciones causales, signos, 
síntomas y tratamiento de la enfermedad no equivale a la aprobación pura y simple de las explicaciones biomédicas.

En Livramento hay un amplio acceso a la información de que la malaria es causada por la picadura de mosquito, pese a que no se comprenda a detalle cómo podría ocurrir. Las directrices relativas al papel del mosquito en la transmisión de la malaria se difunden a partir de los servicios de salud, pero no parecen generar un convencimiento pleno de la población sobre su veracidad.

Así, se producen nuevas explicaciones sobre la ocurrencia de la enfermedad, sin que se nieguen las vehiculadas por el sistema médico oficial. Una de las más relevantes es la reinterpretación de la causalidad, que asocia la ocurrencia de malaria a la suciedad ingerida en el agua o acumulada en el intestino. En el modo de interpretación vigente en Livramento, el mosquito deposita huevos en el agua potable, en la que se desarrollan sus larvas, que son ingeridas por las personas que adquieren así la malaria. En ese caso, el riesgo de contraer la enfermedad sería mayor entre las familias consideradas descuidadas, es decir, con poco cuidado en la limpieza de las fuentes de agua, por dejarlas descubiertas. La misma exposición ocurriría entre las que depositan basura en sus proximidades.

La participación de los mosquitos en la transmisión de la malaria también tiene aspectos controversiales. Los habitantes de Livramento no diferencian el mosquito del género Anopheles —al que el modelo biomédico atribuye el papel exclusivo de la transmisión de la malaria- del zancudo común —Culex quinquefasciatus-, conocido en el lenguaje regional amazónico como carapanã. Según los entomólogos, el carapanã doméstico vuela sólo por la noche y no participa en la transmisión de la malaria. En contrapartida, el Anopheles, implicado en la transmisión de la enfermedad, tiene hábitos de vuelo al amanecer y el atardecer.

En Livramento no se reconoce la diferencia entre estos tipos de mosquito ni las singularidades físicas y comportamentales de los géneros y especies. En consecuencia, se entiende que las estrategias de protección contra las picaduras de los mosquitos deben orientarse al periodo nocturno, el momento de mayor incomodidad por las picaduras de carapanã que, desde el punto de vista biomédico, no interviene en la transmisión de la malaria. En síntesis, la participación de mosquitos en la transmisión de la enfermedad es reconocida y aceptada; sin embargo, no se consideran las diferencias entre las especies de mosquito, sus hábitos, formas y momentos de participación en la transmisión de la malaria y otras enfermedades vectoriales.

Estos detalles llevan a las personas a identificar contradicciones en las explicaciones causales biomédicas, ya que observan periodos del año en que hay elevada ocurrencia de malaria, pero una cantidad insignificante de carapanã. Situaciones como ésta amplían la convicción de que la enfermedad por malaria se debe a otras causas, que trascienden la picadura del mosquito.

El modelo explicativo elaborado en Livramento sugiere que la reconstrucción comunal del modelo de causalidad de la malaria se apoyó en correlaciones establecidas entre: 1) las explicaciones biomédicas sobre la transmisión de parasitosis intestinales y otros agentes que generan enfermedades diarreicas; en ese ámbito, sobresale la recomendación de higienizar el agua para beber; 2) las campañas de control de la malaria destinadas a destruir los criaderos de mosquitos transmisores; 3) las campañas de lucha contra el dengue orientadas a eliminar las larvas de mosquito de los depósitos de agua estancada; 4) la convivencia cotidiana con la malaria, que muestra que la enfermedad afecta el apetito, hincha el abdomen y dificulta la digestión, lo que refuerza la noción de que, más allá de la fiebre, la malaria es una enfermedad digestiva, con mayor expresión en el vientre, y 5) el discurso higienista, fuertemente impregnado en las acciones y recomendaciones de los equipos de salud, que se refleja de manera 
efectiva en la idea de que ingerir "suciedad" puede causar malaria. Lo que se entiende por suciedad son las larvas de mosquitos en el agua y la presencia de residuos sólidos aparentes que de manera ocasional se depositan en los pozos de agua al momento de recolectar y en el almacenamiento inadecuado del agua para consumo humano, pues la localidad no cuenta con agua canalizada.

La lógica subyacente en el modelo de autoatención producido en Livramento sitúa el agua para beber como uno de los elementos primordiales en la preservación o alteración de la salud. Al mismo tiempo, se reinterpretan las informaciones biomédicas sobre la causalidad de las enfermedades que circulan entre la población para adquirir sentidos congruentes con la experiencia cotidiana de enfermedad y con los modos de vida de las personas. Lejos de expresar el desconocimiento sobre los acontecimientos relacionados con la enfermedad, estas narrativas deben entenderse como rechazo a reducir un problema de salud tan relevante para la vida de las personas a meros síntomas de fiebre, temblor y malestar.

Las medidas oficiales recomendadas para prevenir la malaria oscilan entre evitar la exposición a las picaduras y establecer barreras entre las personas y los mosquitos, en particular en los horarios en que los profesionales de la salud consideran de mayor riesgo, es decir, el amanecer y el atardecer, horarios preferenciales de vuelo del mosquito vector de la malaria. Aunque en apariencia simples y fáciles, estas recomendaciones se consideran imposibles de cumplir, porque hacen inviable la realización de tareas de la vida diaria.

El atardecer es el horario de retorno de las actividades laborales, en el que se realiza buena parte de las actividades domésticas o de ocio y cuando la gente se baña en el río o en baños a cielo abierto en las proximidades de las viviendas, pues el agua potable, los baños y las lavanderías cerradas son recursos de los que no se dispone en la localidad. Estas circunstancias hacen que sea casi imposible evitar el contacto con los mosquitos en los horarios prescritos. La gente está aún ocupada en los quehaceres domésticos y los niños juegan en las cercanías de las casas. Las ropas y los utensilios domésticos se lavan por la mañana y al atardecer a la orilla del río o en los pozos poco profundos de construcción artesanal, lo que propicia la exposición a los mosquitos.

Otra medida poco acogida fue el uso de mosquiteros impregnados con insecticida. $\mathrm{Al}$ atardecer aún persiste el calor intenso, típico de los trópicos, y es inviable refugiarse bajo los mosquiteros.

Por último, Livramento es una localidad rodeada de bosque y agua, sin pavimento ni saneamiento básico. El espacio cotidiano de convivencia y trabajo guarda proximidad ineludible con criaderos de insectos. Es decir, vivir es sinónimo de exponerse a la transmisión de la malaria, lo que hace ardua la tarea de su erradicación.

En esas circunstancias, la enfermedad se percibe como un infortunio que no puede evitarse, algo que sucede porque "tiene que suceder", "por suerte": "ahora, como no tenemos cuidado [risas], se nos pega la malaria. Como te dije al principio: nos bañamos y tal, nos da igual, ¿cierto? Entonces, si no nos da [malaria] es incluso por suerte [risas], no se puede decir que es porque no queremos" (entrevista con Milena, 31 años, residente de la sede, comunidad de Livramento, 10 de septiembre de 2009).

La inviabilidad práctica de ejecutar los procedimientos de prevención contra la malaria reubica la ocurrencia de la enfermedad en el nicho de la mala suerte, o bien, expresa un entrecruzamiento entre la causalidad biológica y una vagamente cosmológica, poco elaborada, pero efectiva como ruta explicativa y plan orientador de la acción. Este movimiento interpretativo retira la posibilidad de agencia del sujeto, cuya representación es de impotencia ante el infortunio: "icreo que cuando a uno le toca, le toca!” (entrevista con Josefa, 56 años, residente, igarapé del Mari, 19 de septiembre de 2009). 


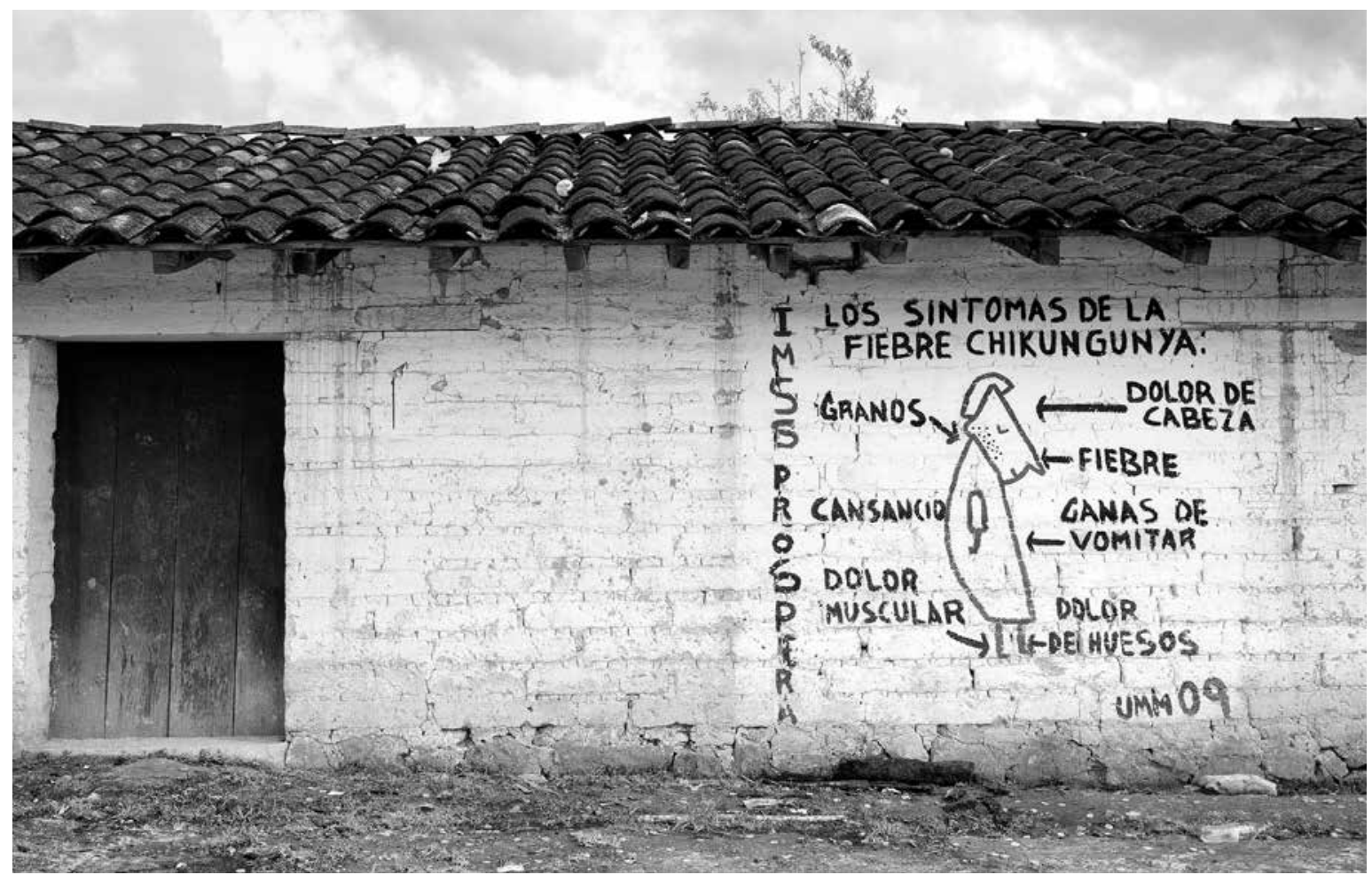

PROMEteo LUCERo - Campañas de salud en las calles principales de Cochoapa el Grande, Guerrero, el municipio con mayor índice de pobreza en el país, con una gran proporción de población tu'un savi -mixteca-. Noviembre de 2015.

La mala suerte es una atribución muy difundida entre las personas que entrevistamos en Livramento. Puede interpretarse como un modo de lidiar con acontecimientos que no se gobiernan, para los cuales los cuidados preventivos disponibles no son efectivos, pues son incongruentes con las condiciones de vida en el área rural.

Si la malaria es un hecho fortuito, imprevisible e inevitable, la obediencia de hábitos ancestrales de las culturas indígenas locales, que prescriben la limpieza diaria en el baño en el río — reforzada, sin duda, por el discurso higienista procedente del contacto recurrente con los servicios de salud-, gana preponderancia, dado que los códigos culturales tradicionales dicen que las enfermedades o infortunios, incluso los más graves, pueden derivarse de la ausencia de limpieza corporal (Gazinelli et al., 2008; Garnelo, 2007).

\section{Estados liminares en la ocurrencia de malaria}

Más allá del modelo biomédico, que opera como fuente de información sobre la malaria, se verificó la existencia de un conjunto de conocimientos ligado a la percepción de las manifestaciones corporales atribuidas a la enfermedad, que produce un concepto propio de estar enfermo. Por tratarse de un área endémica, la mayoría de los habitantes de Livramento ha padecido malaria o ha estado cerca de alguien que vivió la enfermedad varias veces. Por lo tanto, reconocen su afectación en el cuerpo propio o en las evidencias narradas por parientes y amigos. Sin embargo, describen un modo de evolución de la enfermedad cuyas fases son distintas de las que la nosología médica entiende como verdaderas. 
Algunas manifestaciones corporales, como indisposición, "peso en la cabeza" y frío, demarcan la presencia de la malaria en el cuerpo, pero para los habitantes de Livramento no equivalen a estar enfermo todavía. Se trata de un estado liminar en el que la persona puede volver a la condición sana o evolucionar a la de enfermo, según la "fuerza" de la enfermedad, la capacidad corporal para reaccionar a ella o los cuidados tomados para fortalecerse. En este estado liminar, la persona es clasificada como portadora de "malaria hacia adentro", es decir, está con malaria en el cuerpo, pero los síntomas son poco evidentes y no se establece el papel de enfermo. En Livramento, esta condición se denomina "malaria interna".

El eje que define la condición de enfermedad se desplaza del polo biológico a lo social. En Livramento, la malaria es reconocida como elemento que compromete la capacidad de garantizar la subsistencia, la convivencia con la familia y el ocio. Estar enfermo es estar incapacitado para mantener las actividades laborales rutinarias y ser dispensado de manera temporal de las obligaciones sociales en la comunidad. Algunos estudios publicados en Brasil corroboran la idea de que, entre las clases populares, el demarcador de gravedad de la enfermedad es cuando se presenta la incapacidad para el trabajo y el ocio (Leite y Vasconcellos, 2003; Gonçalves, 2002; Fechinne, Quirino y Paiva, 2012).

El cuerpo se entiende como un importante instrumento de relación del individuo con el mundo. En este ámbito, las dimensiones social y biológica de la existencia son indisociables y su preservación está, sin embargo, amenazada por la malaria. Así, la percepción de cualquier tipo de malestar corporal suscita la sospecha de que la malaria se introdujo en el cuerpo. De ahí en adelante, cualquier síntoma que aparezca tiende a asociarse a la enfermedad. Con frecuencia, este movimiento produce un conjunto de manifestaciones corporales, como tos y dolor en la columna, que desde el punto de vista biomédico no guardan relación con la malaria, pero que son reconocidas por las personas en Livramento como parte de una constelación sintomática que empuja al portador a salir de la situación liminar y moverse hacia el polo de la enfermedad.

La aparente incongruencia de los síntomas atribuibles a la malaria se vincula a su reconocimiento como un hecho social total (Mauss, 2003), pues se manifiesta de manera global, une el plano corporal, objeto de preocupación del modelo biomédico, al plano social, en el que repercute de manera negativa en las estrategias de mantenimiento de la vida.

Portar la malaria en el cuerpo coloca al individuo en un estado de alerta, justificado por la inminente aparición de los síntomas incapacitantes de verdad, como la fiebre alta, fuertes dolores de cabeza y temblores. En esa fase, identificamos diversas actitudes posibles, cuya adopción dependerá de la situación momentánea de vida de la persona, su red de cuidados o la propia estructura del servicio de salud disponible en la localidad. Una de las alternativas es buscar atención biomédica en el servicio de salud. También se puede optar por la autoatención, con o sin uso de los recursos terapéuticos del sistema oficial de salud.

Es importante resaltar que la opción de la automedicación no equivale a rechazar el tratamiento oficial. Por el contrario, se entiende que la persona tiene malaria y es necesario tratarla. Sin embargo, los condicionantes de la vida personal y de su red de cuidados deben ajustarse a las opciones ofrecidas por la red de servicios de salud. Se entiende que la adhesión al tratamiento de la medicina oficial equivale también a aceptar el rótulo de enfermo de malaria, así como las modificaciones que la adopción de este papel imprime en la vida cotidiana. Es decir, la búsqueda de curación tiene, entre sus consecuencias, aceptar el reconocimiento social de la condición de enfermo que promueve una suspensión temporal de las actividades de subsistencia y otras atribuciones cotidianas. 
Ante la sospecha de malaria, los habitantes fueron enfáticos al señalar la realización del examen de detección de Plasmodium como la primera acción. El modelo de autoatención practicado en Livramento también demuestra la apropiación del modelo explicativo biomédico que alimenta buena parte de las ideas locales sobre el proceso de enfermedad por malaria. Aunque el diagnóstico de malaria se construye a partir de una experiencia colectiva del cuerpo enfermo, no se descarta la realización del examen ofrecido por el servicio oficial de salud. El examen, en realidad, se considera indispensable para confirmar el diagnóstico, además de que es la vía que permite la obtención del tratamiento específico, puesto a disposición sin costo por los servicios de salud.

Sin embargo, si el uso del medicamento se considera esencial, seguir los esquemas terapéuticos oficiales prescritos por los profesionales de salud, no. De hecho, esas prescripciones se adaptan para hacerlas congruentes con la lógica del modelo de autoatención vigente en Livramento, que sugiere un protagonismo interesado en ampliar el control sobre las estrategias de curación, los cuerpos y las vidas.

Así como la búsqueda de la curación, el entendimiento sobre los modos en que actúan los tratamientos de malaria también está marcado por una fuerte ambigüedad. Una de las razones es el entendimiento de que bajo la acción del medicamento la malaria es enviada "hacia fuera" del cuerpo, para hacer explícita la constelación sintomática, hasta entonces poco perceptible. La externalización de los síntomas demarca la instalación efectiva de la enfermedad en el cuerpo de la persona, en consecuencia, altera su estatus hacia el de alguien que necesita cuidados y no es capaz de realizar las obligaciones cotidianas necesarias para garantizar la subsistencia.

Puesto que la búsqueda del tratamiento oficial implica un encuadramiento en el papel de enfermo, en algunas situaciones el portador de malaria interna intentará postergar el diagnóstico y el tratamiento para tratar de completar sus tareas pendientes antes de aceptar el cambio de estatus en la comunidad. Estas situaciones son evidentes en particular en tiempos de intenso trabajo agrícola, como en las épocas de siembra o de procesar los productos cosechados, cuyos momentos de ejecución son precisos y están determinados por los ciclos de la naturaleza, y no pueden adelantarse o postergarse con facilidad.

Para obtener este resultado, los residentes utilizan un repertorio de medicamentos a los que llaman remedios calmantes. Por lo general, son analgésicos y antitérmicos de bajo costo, adquiridos sin prescripción médica incluso en los pequeños comercios de la localidad. Su finalidad es suavizar los síntomas de la malaria, en especial la fiebre y los dolores, mientras la persona posterga el comienzo del tratamiento con antimalárico.

El uso de antimaláricos en el régimen de automedicación también puede ocurrir en estos casos, para obtener un efecto calmante. Por ejemplo, Andressa, de 15 años de edad, habitante de una localidad alejada del núcleo central de Livramento, cuando sospechó que estaba enferma de malaria, pensó en ir al puesto de salud. Sin embargo, era tarde de viernes y la unidad de salud no funciona ese día ni en ese horario. Además, todos en su casa estaban ocupados en una actividad de subsistencia considerada inaplazable.

La familia optó por utilizar sus reservas de antimaláricos disponibles en casa y administrárselas a Andressa. Estas reservas domésticas son los sobrantes de tratamientos de personas que no los concluyen o toman dosis menores de las recetadas por los servicios de salud. Es decir, en ese ámbito también hay una reinterpretación de las prescripciones biomédicas, de acuerdo con la lógica explicativa del sistema de autoatención vigente en Livramento. Andressa empezó el tratamiento con subdosis de antimaláricos mientras aguardaba la reapertura de la unidad de salud el lunes siguiente.

En términos físicos, la aparición de la fiebre y los temblores se establece como demarcador 
objetivo de que la persona migró de la condición liminar hacia la de enfermo de malaria. En varios testimonios, los picos de fiebre y temblor fueron representados como la propia malaria, con expresiones como "cuando viene la malaria" o "cuando la malaria ataca". La eclosión de esos signos reubica a la persona en el nivel de los que necesitan cuidados específicos para su condición y de alguien que está liberado del trabajo cotidiano y las obligaciones de reciprocidad que rigen la vida comunitaria. En oposición, la persona debe soportar el tratamiento con estoicismo y aceptar la restricción de su autonomía en la toma de decisiones, en particular en lo que se refiere a la aceptación de las terapéuticas instituidas.

Gonçalves (2002) identificó como elemento inherente al papel social de enfermo el establecimiento de cuidados específicos, distintos de las conductas adoptadas en el día a día del ambiente familiar. La oferta de esos cuidados ocurre de manera simultánea a una suspensión temporal del derecho a tomar ciertas decisiones en lo que se refiere al bienestar, el ocio y la dieta. También forma parte de la condición de enfermo ser monitoreado de cerca por los miembros de la red de cuidados que se establece con la finalidad de tratarlo. En suma, es una reducción o supresión temporal de la condición de adulto y miembro pleno del grupo.

Además de las manifestaciones características de la malaria, el individuo en tratamiento también puede presentar otros signos y síntomas, como dolor en el estómago, vómito y alergias, atribuidos por el sistema oficial de salud a efectos colaterales de los medicamentos. Sin embargo, en la interpretación de los residentes de Livramento, estos síntomas

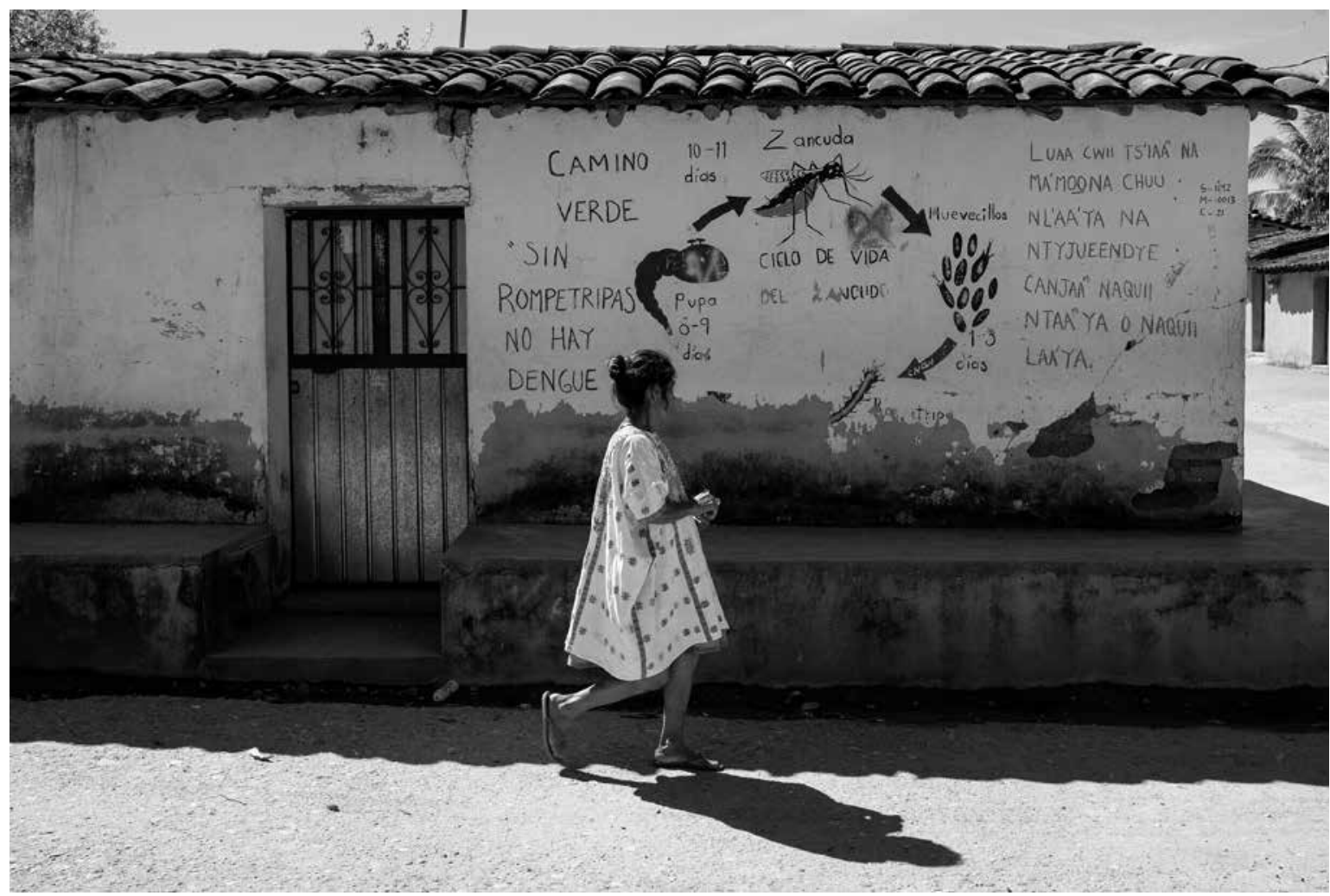

Prometeo Lucero - Campaña contra el dengue en las calles de Xochistlahuaca, Guerrero, municipio con población indígena amuzga. Diciembre de 2015. 
son algo esperado y se entiende como intrínsecos a las manifestaciones de la malaria, pues no hay distinción entre la expresión sintomática de la enfermedad, producida por la acción del parásito, y las relativas al efecto indeseado de los medicamentos.

La aparición de efectos colaterales de los antimaláricos, como alergias con picazón y ampollas, es relativamente común, pero en Livramento estos síntomas se consideran manifestaciones de cura, en las cual el remedio está "botando la malaria para afuera" del cuerpo. Estas reacciones se atribuyen al carácter "fuerte" del medicamento, visto como capaz de vencer y expulsar la enfermedad; no se identifican como un efecto secundario no deseado y sí como una ocurrencia positiva que anuncia la curación en un futuro inmediato.

Los recursos terapéuticos oficiales no están despojados de ambigüedad. Hay una idea clara de que son capaces de curar, pero su actuación también daña de forma grave y muchas veces irremediable el organismo del enfermo. En esas circunstancias, se entiende que los daños causados por el antimalárico deben ser atenuados para que el paciente llegue a una cura completa. Para ese fin, se adoptan los llamados "remedios auxiliares", que pueden ser tanto caseros como industrializados, cuya función es ayudar al cuerpo a soportar la agresividad del tratamiento oficial para la malaria.

Los remedios auxiliares establecen un contrapunto que equilibra y neutraliza, al menos en parte, la peligrosidad de los antimaláricos, pues tienen como objetivo reducir los efectos negativos del tratamiento sobre el aparato digestivo y la pérdida de fuerzas, quejas comunes de los enfermos de malaria. En ese repertorio terapéutico se incluyen tanto los de fabricación doméstica, como los tés de la herbolaria tradicional amazónica, como medicamentos alopáticos o incluso fitoterapéuticos industrializados.

La adopción de los medicamentos auxiliares suele estar orientada por funcionarios de farmacias y por consejos de personas que han sufrido de malaria o cuidaron a un pariente en esas condiciones y obtuvieron una experiencia positiva con ese procedimiento.

Para los entrevistados, los medicamentos auxiliares, además de que no provocan efectos indeseables, favorecen una rápida recuperación. A varios se les atribuye la capacidad de "fortificar la sangre y desinflamar los órganos" más afectados por la malaria, como el estómago y el hígado, órganos que las personas de Livramento identifican como los lugares de las principales quejas de los enfermos. Otro efecto valorado es la ayuda para el retorno del apetito del paciente.

Aquí la automedicación se efectúa a partir de un principio de complementariedad en el cual los remedios calmantes y auxiliares, así como el tratamiento con antimalárico, más que actuar sobre una u otra fase de la enfermedad, se entienden como recursos terapéuticos complementarios entre sí, todos necesarios por igual para la cura.

\section{La malaria "mal curada": criterios de cura según el modelo de autoatención en Livramento}

Según la lógica totalizante en la interpretación de la salud y la enfermedad, la condición de enfermo no se acaba con el alta dada por el servicio de salud. La cura se confirma con la ausencia del parásito plasmodium en la sangre, por medio del examen denominado lámina de verificación de cura (LVC). No obstante, después del alta, los habitantes de Livramento consideran que la malaria permanece en el interior del cuerpo y produce impurezas y un estado de "debilidad" que en cualquier momento puede reinstalar el estado de enfermedad.

En este sentido, la malaria puede haber "perdido la fuerza" y dejado de intervenir en el desempeño social de la persona afectada. No obstante, la desaparición de los marcadores corporales más evidentes de la enfermedad no se reconoce como 
una condición suficiente para garantizar que alguien está curado. Para explicar estas situaciones, se dice que la malaria ha retomado su estado liminar y se ha guardado "hacia dentro" del cuerpo.

Este hecho es corroborado por la presencia de síntomas, ampliamente descritos por los enfermos, como la sensación de hinchazón en la barriga, malestar general, debilidad física y otras quejas igual de vagas que pueden durar por varios meses:

Después de una semana [del final del tratamiento biomédico], usted hace lámina de nuevo. Incluso si da negativo, la persona no está 100\% curada. Todavía siente los efectos negativos de la enfermedad y tiene que seguir tomando el remedio casero para evitar que la enfermedad [la malaria] quede en los órganos que más le gusta: el hígado y el bazo. Cuando pasan los síntomas, usted piensa que está curado, pero no; [la malaria] continúa ahí y puede volver a crecer. Así, si tiene ese remedio que usted siente que le hace bien, mejor se lo sigue tomando (entrevista con Jose Tavares, 56 años de edad, residente de la sede, comunidad de Livramento, 11 de septiembre de 2009).

Según el modelo de autoatención vigente en Livramento, la condición de portador de la malaria interna perpetúa ciertas restricciones y prescripciones adecuadas para el estado liminar, aunque el tratamiento con antimalárico haya concluido de forma eficaz y suprimido los signos clínicos más agudos e incapacitantes de la enfermedad.

Los habitantes de Livramento distinguen entre el criterio de curación adoptado por los servicios de salud y el instituido en el sistema local de autoatención: lo que el modelo biomédico considera como cura, en las prácticas de autoatención de Livramento se percibe como una etapa intermedia entre la enfermedad y la cura, y es necesario continuar los cuidados que el modelo biomédico desconoce o ignora y que es incapaz de suplir.
La percepción de que los síntomas marcadores de la enfermedad desaparecen después del uso de medicación oficial se sintetiza en la expresión "estar bien" después de la agresión perpetrada por la malaria. Pero en la jerga de Livramento "estar bien" no equivale a "estar curado".

Para el conocimiento local, la malaria perjudica el proceso digestivo por las lesiones que causa al hígado y el estómago, entonces se establece la demanda de que estos órganos sean tratados. Este paso se considera importante para hacer viable la curación y acelerarla. Según la tradición oral, la malaria mal curada se convierte en otras enfermedades más graves, como hepatitis y leucemia. Estas afirmaciones se basan en la rememoración de casos, presentados como situaciones reales, de moradores que desarrollaron esas patologías como consecuencia de la curación incompleta de la malaria. De acuerdo con estos relatos, algunos de los enfermos habrían evolucionado hacia la muerte por no adoptar los cuidados necesarios para la recuperación del hígado y el bazo.

Entre las facetas del sistema de autoatención en Livramento, la cuestión alimentaria surge como una de las medidas de defensa y fortalecimiento corporal más importantes para evitar que la malaria se instale y se torne crónica después de instalada.

El hilo conductor de la dieta del enfermo de malaria es que tanto la enfermedad como el medicamento utilizado para tratarla perjudican la digestión. Los juicios son congruentes con el entendimiento local de que la malaria se asienta y lesiona órganos considerados por los informantes como integrantes del sistema digestivo, en específico el hígado, el bazo y el estómago, lo que resulta en dolor abdominal, abdomen dilatado, diarrea, falta de apetito y mala digestión.

Cuando la persona es socialmente reconocida como portadora de malaria hacia adentro, se adoptan muchas restricciones en el menú y se da prioridad a los alimentos que no amplían el riesgo de pasar del estado liminar a la condición plena de 
enfermo. En estas circunstancias, se ponen en acción los cuidados dietéticos especiales que favorecen el buen funcionamiento de estos órganos. Se instala la restricción de grasas, alimentos ácidos y reimosos.

Reima es un término regional, vinculado a las cosmologías indígenas, utilizado para expresar relaciones de hostilidad de sujetos no humanos, como los peces y la carne de cacería, que causan enfermedades como reacción a la depredación humana. Es así que las personas enfermas o en situación liminar deben evitar ciertos alimentos capaces de agravar su condición (Garnelo, 2007).

En el caso de la malaria, en Livramento se entiende que una dieta adecuada para personas en situación liminar y enfermas debe restringir el consumo de animales cuya carne grasosa puede causar inflamación y dificultar la cicatrización de heridas en el estómago, el bazo y el hígado debidas al parásito de la malaria. Sumado a ello, la adopción de actitudes ponderadas, el mantenimiento del reposo y el rechazo a los excesos laborales y gastronómicos son medidas que ayudarán al portador de malaria interna a superar la condición liminar y recuperar la salud plena. La combinación de estas medidas también tiene por objeto evitar las recaídas, recurrentes en la localidad.

La condición de cura se refiere a la desaparición completa de los síntomas agudos y característicos de la malaria - en particular la fiebre y los temblores intensos-, del malestar percibido en el interior del abdomen, la sensación de debilidad y la indisposición para el trabajo y otras tareas cotidianas.

No es raro que esas manifestaciones se prolonguen por meses, lo que reafirma la interpretación nativa de que la malaria retrocedió, "se recogió", pero no se ha curado y puede haber una recaída en cualquier momento. En varias situaciones, la persistencia de los síntomas conduce a la conclusión de que la malaria fue mal curada y se ha convertido en un estado crónico, para el cual sería imposible obtener una cura completa. En esos casos, el conjunto de cuidados dirigidos a la alimentación y la protección de los órganos lesionados, que ya no cicatrizan, debe mantenerse de por vida.

Destaca aun la existencia de situaciones en las que apenas persiste el malestar difuso, sin expresión clara de signos o síntomas característicos de la malaria. En estos casos, el portador de la malaria mal curada tiende a quedar prisionero en la condición liminar, con restricciones en su autonomía y capacidad de agencia; persiste también el control más o menos explícito de su grupo familiar, que conduce y perpetúa la red de cuidados que busca garantizar que la condición liminar no evolucione, sin posibilidad de retorno a la condición sana.

Adquirir malaria varias veces seguidas es una de las condiciones reconocidas como propiciadoras de la cronicidad, que imposibilita la cura por el daño permanente de los órganos atacados por el parásito. Es importante notar que gran parte de la población de Livramento ha padecido episodios repetidos de malaria. Es decir, desde su punto de vista, la asignación de cronicidad a los miembros de la comunidad es un evento plausible en el escenario de eventos en un futuro próximo.

\section{Juicios e interfaces con los agentes oficiales de cura}

Los habitantes de Livramento tienen contacto regular con profesionales de la salud que efectúan acciones para el control de la malaria. En esta interacción construyeron relaciones de profunda desconfianza en cuanto a la calidad y efectividad de los procedimientos ofrecidos. Los profesionales son, por regla general, personal técnico de nivel medio - agentes de endemias-, entrenado para efectuar procedimientos estandarizados de diagnóstico y tratamiento. Dada la proximidad con la capital, los agentes de endemias, que eran muy jóvenes en la época de la recolección de datos, se desplazaban a diario a Livramento, pues no residían allí. Su juventud y su 
forma risueña, despreocupada y juguetona de comportarse eran motivo de críticas severas por parte de la población, que consideraba inadecuadas sus actitudes, carentes de la serenidad y prudencia que se espera de un profesional de la salud. La censura se dirigía en particular a uno de los agentes de endemias que oía una pequeña radio de pila y bailaba mientras trabajaba, lo que agravaba los juicios negativos sobre su comportamiento, considerado inadecuado para las funciones que ejercía.

Los agentes de endemias gozan de bastante autonomía y no tienen jefes inmediatos a quienes rendir cuentas del cumplimiento de sus actividades rutinarias. Un punto destacado en sus tareas era el desplazamiento intermitente en un bote de aluminio con motor para buscar casos, lo que resultaba en un tránsito repetido entre el aglomerado central de domicilios y la periferia de Livramento. Este movimiento, sumado a los viajes frecuentes a Manaos en horarios laborales, acentuaba las reacciones de desconfianza, pues se percibía que los agentes de endemias estaban "paseando" y gastando combustible de manera innecesaria.

Las personas también desconfían de la calidad de las láminas recolectadas para diagnosticar y verificar la cura. Asimismo, critican la capacitación de los agentes de endemias. Los consideran poco escolarizados y han coleccionado historias — contadas en las familias y a veces para plateas mayores - de intercambio de láminas por fallas en la identificación de los examinados, errores de diagnóstico y en la prescripción de la dosificación del antimalárico, y sobre todo, descuido en la realización de las tareas.

Los errores derivados de la lectura incorrecta de láminas para diagnóstico de malaria son temidos en Livramento, pues pueden implicar un retardo del diagnóstico correcto del tipo de malaria u otro tipo de afección, y colocar al enfermo en riesgo de empeorar y de muerte, dada la rapidez con que la enfermedad toma el cuerpo de un individuo.
Los técnicos de endemias también se consideran irrespetuosos con las mujeres jóvenes y las reglas de etiqueta alimentaria vigentes en la comunidad cuando critican o llegan a pedir comida en las casas. La falta de vínculo, el desinterés y la negligencia, así como el irrespeto sistemático de las normas de sociabilidad comunal, restan valor a la imagen cuidadora que las personas de Livramento desean ver en los agentes del poder público.

Más allá de la poca confianza depositada en la calidad del trabajo y las actitudes de los agentes de endemias, se verifica que éstos quebrantan los valores y patrones de comportamiento considerados adecuados en Livramento, donde se espera una circunspección que los agentes de endemias no cultivan. La ocurrencia de errores de diagnóstico y en la identificación de destinatarios del tratamiento refuerza la idea de que los agentes no poseen la calificación necesaria para el trabajo, pero, sobre todo, de que no toman en serio su trabajo ni el sufrimiento de los enfermos a quienes atienden. Dificultades ocasionales en el tratamiento, incluso las que pueden atribuirse a las relecturas de los usuarios de las prescripciones, refuerzan la conclusión de que los agentes de endemias tienen una utilidad limitada en la comunidad.

Las interacciones entre la población, los agentes del poder público y las acciones orientadas al control de la malaria también están impregnadas por las desigualdades sociales que se perpetúan en la localidad. Esta situación se ejemplifica con la campaña de distribución de mosquiteros impregnados de repelente. La iniciativa fue dirigida por la Fundación de Vigilancia en Salud de Amazonas (FVS-AM), con sede en la capital, responsable del control de endemias en el estado.

Para hacer viable la distribución de los mosquiteros impregnados, la FVS-AM estableció una sociedad con la Asociación de Vecinos de Livramento y le asignó las tareas de identificar y registrar a las familias en situación de mayor riesgo de transmisión de la enfermedad y seleccionar a los beneficiarios prioritarios. 


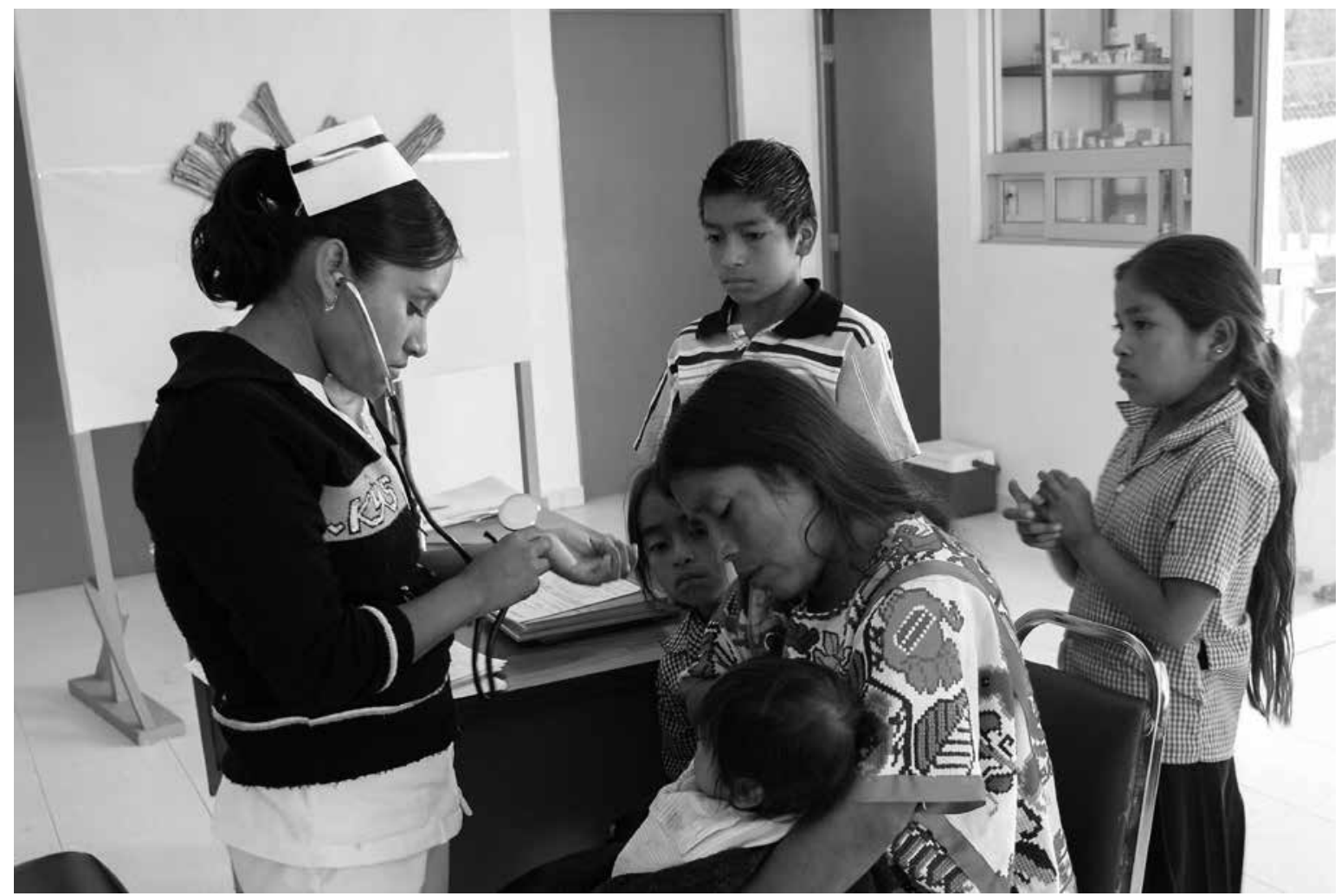

Prometeo Lucero - Centro de Salud en Cochoapa el Grande, Guerrero. Octubre de 2009.

La expectativa de la FVS-AM era que la distribución estuviera pautada por criterios técnicos, relativos a la concentración de beneficiarios en lugares de mayor exposición a los criaderos y el riesgo de transmisión. Los parámetros que orientaron a la Asociación de Vecinos en la distribución de los mosquiteros se basaron, en cambio, en el parentesco y las alianzas políticas entre las familias. Así, los residentes de pequeños asentamientos con fuerte incidencia de malaria fueron excluidos de la lista de beneficiarios y se dio prioridad a los habitantes de la sede de la comunidad de Livramento, donde hay un riesgo menor de transmisión de malaria, pero donde residen las familias de más prestigio social. En este caso, las pautas que rigen la sociabilidad entre los grupos familiares de Livramento preponderaron sobre las razones técnicas, y la entrega de mosquiteros se entendió más como una ayuda social para aquellos a quienes se deben obligaciones de parentesco o reciprocidad, que como una técnica de barrera dirigida a interrumpir la transmisión de la malaria.

Debido a la frágil interacción entre los profesionales de la salud y la población de Livramento, no hay un diálogo entre las interpretaciones sobre el proceso salud/enfermedad/atención instituidas en torno a la malaria que permita la compresión mutua entre los representantes de ambos mundos. La población de Livramento es proactiva en el mantenimiento de sus concepciones de enfermedad, así como de los valores y actitudes que rodean el cuidado, y se preocupan por velarlos a los ojos de los profesionales de salud. Éstos, a su vez, tienden a guardar la convicción de ser los únicos portadores de las interpretaciones válidas del proceso salud/enfermedad/atención. 


\section{Consideraciones finales}

Para Menéndez (2016), las relaciones entre culturas siempre se producen de modo intercultural. Es decir, sus sujetos intercambian valores, bienes e ideas que transitan entre los polos de la relación y estos intercambios pueden ser bastante asimétricos, dadas las condiciones estructurales de desigualdad social vigentes a escala planetaria.

Las concepciones y prácticas de enfermedad, cura y cuidados de grupos subalternos, como los observados en Livramento, están directamente relacionadas con sus condiciones de vida y confieren la posibilidad, aunque momentánea, de controlar el rumbo de su existencia y manejar los acontecimientos y desenlaces de sus problemas de salud (Alves y Souza, 1999).

A lo largo de las décadas, la población de Livramento ha elaborado un cuerpo teórico explicativo del proceso salud/enfermedad/atención, capaz de propiciar modos de enfrentar sus problemas de salud y de dialogar, adoptar, ignorar o reinterpretar los conocimientos y prescripciones biomédicas que les son accesibles. No obstante, lo hacen en sus propios términos, pues anhelan tener el mayor control posible de los recursos preventivos, terapéuticos y económicos a su alcance, los cuales expresan categorías de pensamiento y actitudes adoptadas en la praxis del día a día por personas que viven en íntimo y prolongado contacto con la malaria.
La diversidad de explicaciones causales de la población de Livramento no pretende excluir modelos explicativos sino acomodar las múltiples vías de interpretación y acción para que favorezcan la intervención en sus problemas de salud. Los modelos biomédicos de intervención no se descartan, sino que se rearticulan e incluso se reconstruyen para producir un nuevo cuadro de significados socialmente compartidos, mediante los cuales las familias los hacen más coherentes con sus necesidades y congruentes con los modos de vida cotidianos.

Entre las limitaciones del estudio, sobresale la selección de Livramento y de los informantes por muestreo de conveniencia. Sin embargo, el artículo no objeta la producción de un perfil epidemiológico de carácter representativo de la situación de la malaria en la región. El carácter cualitativo del estudio se enfoca en entender los sentidos atribuidos al cuadro de convalecencia por malaria y las estrategias de cura y cuidados disponibles en el lugar, porque se entienden como producciones culturales relevantes para orientar las políticas públicas destinadas a controlar esta enfermedad.

El entendimiento de las estrategias culturales de producción, consumo y circulación de saberes y prácticas, como las encontradas en Livramento, es fundamental para alcanzar un proyecto humanizado y técnicamente calificado para el tratamiento, por parte de las políticas públicas, de los problemas de salud que se reproducen en los rincones de la Amazonia. D

\section{Bibliografía}

Adams, Cristina, Rui Murrieta y Walter Neves, 2006, “Introdução”, en Cristina Adams, Rui Murrieta y Walter Neves (orgs.), Sociedades caboclas amazônicas. Modernidade e invisibilidade, Fundação de Amparo à Pesquisa do Estado de São Paulo/Anablume, São Paulo, pp. 15-32.

Alves, Paulo César y lara Maria A. Souza, 1999, "Escolha e avaliação de tratamento para problemas de saúde. Considerações sobre o itinerário terapêutico", en Maria Cristina Rabelo, Paulo César Alves y lara Maria A. Souza (orgs.), Experiência de doença e narrativa, Fundação Oswaldo Cruz, Río de Janeiro, pp. 125-138.

Braz, Rui Moreira, Elisabeth Carmen Duarte y Pedro Luiz Tauil, 2013, "Caracterização das epidemias de malária nos municípios da Amazônia Brasileira em 2010", en Cadernos de Saúde Pública, vol. 29, núm. 5, pp. 935-944. 
Buchillet, Dominique, 1991, “A antropologia da doença e os sistemas oficiais de saúde”, en Dominique Buchillet, Medicinas tradicionais e medicina ocidental na Amazônia, Museu Paraense Emílio Goeldi/Editora Cejup/Universidade do Estado de Pará, Belém, pp. 21-44.

Canelas, Tiago, Carlos Castillo-Salgado y Helena Ribeiro, 2018, "Analyzing the Local Epidemiological Profile of Malaria Transmission in the Brazilian Amazon between 2010 and 2015", en PLos, Currents Outbreaks, 27 de marzo, edición 1. Disponible en línea: <http://currents. plos.org/outbreaks/article/analyzing-the-local-epidemiological-profile-of-malaria-transmission-in-the-brazilian-amazon-between2010-and-2015/>.

Fechine, Juliana, Glauberto Quirino y Dafne Paiva, 2012, "Percepçã das puérperas quanto aos cuidados prestados pela equipe de saúde no puerpério", en Revista da Rede de Enfermagem do Nordeste, vol. 13, núm. 1, pp. 74-84. Disponible en línea: <http://www.redalyc.org/ articulo.oa?id=324027980010>.

Garnelo, Luiza, 2007, “Cosmologia, ambiente e saúde: mitos e ritos alimentares Baniwa”, en História, Ciências, Saúde-Manguinhos, vol. 14, supl., pp. 191-212.

Garnelo, Luiza y Sully Sampaio, 2005, "Globalização e ambientalismo: etnicidades polifônicas na Amazônia”, en História, Ciências, SaúdeManguinhos, vol. 12, núm.3, pp. 755-768.

Gazinelli, Maria Flávia Carvalho, Helmut Kloos, Rita de Cássia Marques, Dener Carlos dos Reis y Andrea Gazzinelli, 2008, "Popular Beliefs about the Infectivity of Water among School Children in Two Hyperendemic Schistosomiasis Areas of Brazil”, en Acta Tropica, vol. 108, núm. 2, pp. 202-208.

Gonçalves, Helen, 2002, A peste branca. Um estudo antropológico sobre a tuberculose, Universidade Federal do Rio Grande do Sul, Porto Alegre.

Katsuragawa, Tony Hiroshi, Luiz Herman Soares Gil, Mauro Shugiro Tada y Luiz Hildebrando Pereira da Silva, 2008, "Endemias e epidemias na Amazônia: malária e doenças emergentes em áreas ribeirinhas do Rio Madeira. Um caso de escola”, en Estudos Avançados, vol. 22, núm. 64, pp. 111-141.

Leite, Silvana Nair y Maria da Penha Costa Vasconcellos, 2003, "Adesão à terapêutica medicamentosa: elementos para a discussão de conceitos e pressupostos adotados na literatura”, en Ciência e Saúde Coletiva, vol. 8, núm. 3, pp. 775-782.

Mauss, Marcel, 2003, Sociologia e antropologia, Cosac \& Naify, São Paulo.

Menéndez, Eduardo, 2003, "Modelos de atención de los padecimientos: de exclusiones teóricas y articulaciones prácticas", en Ciência \& Saúde Coletiva, vol. 8, núm. 1, pp. 185-208.

2016, "Salud intercultural: propuestas, acciones y fracasos", en Ciencia \& Saude Coletiva, vol. 21, núm. 1, pp. 109-118.

Ministério da Saúde, 2003, Programa nacional de prevenção e controle da malária (PNCM), Editora do Ministério da Saúde, Brasilia.

—_, 2015, "Situação epidemiológica da malária no Brasil, 2012 e 2013”, en Boletim Epidemiológico, vol. 46, núm. 43, pp. 1-17. Disponible en línea: <http://portalarquivos2.saude.gov.br/images/pdf/2015/dezembro/16/2015-003---Mal--ria.pdf>.

Ministério da Saúde-Secretaria de Vigilância em Saúde, 2017, “Lista de municípios pertencentes às áreas de risco para malária”. Disponible en línea: <http://portalarquivos.saude.gov.br/images/pdf/2017/maio/19/Lista-de-municipios-pertencentes-as-areas-de-risco-ou-endemicas-paramalaria.pdf>.

Organización Panamericana de la Salud (OPS) y Organización Mundial de la Salud (OMS), 2018, Actualización epidemiológica. Aumento de malaria en las Américas, Organización Panamericana de la Salud/Organización Mundial de la Salud, Washington, D. C. Disponible en línea: <http://www.paho.org/hq/index.php?option=com_docman\&task=doc_view\&ltemid=270\&gid=43437\&lang=es>.

Saraiva, Maria das Graças Gomes, Raul Diniz Souza Amorim, Marco Antônio Sabóia Moura, Flor Ernestina Martinez-Espinosa y Maria das Graças Vale Barbosa, 2009, "Expansão urbana e distribuição espacial da malária no município de Manaus, Estado do Amazonas”, en Revista da Sociedade Brasileira de Medicina Tropical, vol. 42, núm. 5, pp. 515-522.

\section{Entrevistas}

Milena, comunidad de Livramento, 10 de septiembre de 2009.

Josefa, comunidad del igarapé del Mari, 19 de septiembre de 2009.

José Tavares, comunidad de Livramento, 11 de septiembre de 2009. 